\title{
Evaluation of Fault Voltage and Current in a Symmetric Power System Network
}

\author{
K.Seetharamayya ${ }^{1}$, M.Venkateswara Rao ${ }^{2}$ \\ P.G Student, EEE, GMRIT, Rajam, India ${ }^{1}$ \\ Associate Professor, GMRIT, Rajam, India ${ }^{2}$
}

\begin{abstract}
The subject of Evaluation of Fault voltages and currents in a power system network is very important part of power system analysis for stable and economical operations of a Power System. The main objective of the short circuit fault analysis is to simulate short circuit faults on different buses of a power system network and to estimate the state of the power system before and after a fault, which includes various bus voltages and current flow on various transmission lines. The analysis of power systems under faulted condition provides information regarding circuit breaker selection, relay setting, and the stability of the systems operation. Two different MATLAB based programs were written; one program was for Load Flow Studies to determine the pre-fault conditions based on Newton-Raphson method, while the other was for three-phase short-circuit studies. It was observed that the fault currents were mostly excessively high. In this paper, three-phase symmetrical fault was simulated on the standard IEEE 6 bus and IEEE 30 bus system and fault voltage and fault current level is calculated and short circuit MVA rating for the circuit breaker has been chosen.
\end{abstract}

Keywords: Evaluation of fault analysis, NR method, symmetrical fault (LLL) calculation, short circuit MVA.

\section{INTRODUCTION}

The use of Electrical energy increases more and more on a daily basis. Technological development is the reason for the use of electrical energy. Electric power is generated, transmitted and distributed via large interconnected power systems. The generation of electric power takes place in a power plant. Every year many more power stations, transmission lines and substations are constructed. This situation increases the fault current levels in power systems. During normal operating conditions, current will flow through all elements of the electrical power system within pre-designed values which are appropriate to these elements ratings. The fault phenomenon affects system's reliability, security, and energy quality, and can be considered stochastic. In 1956, L.W. Coombe and D. G. Lewis proposed the first fault analysis program. Many exiting texts offer an extensive analysis in fault studies and calculation.[1-3].

The Evaluation of fault analyses is are very important for the power system studies since they provide data such as voltages and currents during and after the various types of faults which are necessary in designing the protective schemes of the power system. Short circuit and protection studies are essential tools for the electric energy system engineer. The main task is to calculate fault conditions and provide protective equipments designed to isolate the faulted zone from the remainder of the system in the appropriate time. And also carefully designed to power system network subjected to damaging high magnitude current during a fault conditions. [4-6]

A Fault is defined as any failure which interferes with the normal current flow. If the insulation of the system fails at any point or if two or more conductors that normally operate with a potential difference come in contact with each other, a short-circuit, or fault, is said to be occur.
Some of the common causes have their origins in natural disturbance like lightning, high speed winds, earthquakes, earth tremors, snow, frost etc. There may be accidental faults such as falling of trees along a line, vehicles colliding with supporting structures, airplane crashing with line. [7-10]

Fault analysis can be broadly grouped into symmetrical (LLL) and unsymmetrical (LG, LL, LLG) faults. A balanced three phase fault occurs when there is a simultaneous short circuit across all three phases. This is called as Symmetrical fault. If only some phases are affected, the resulting Unsymmetrical fault. The effects of faults on power system are: Overheating and mechanical forces developed by faults may damage the electrical equipment such as bus-bars, generators and transformers $\&$ the voltage profile of the system may be reduced to unacceptable limits as a result of fault. [11-16]

The process of evaluating the system voltages and currents under various types of short-circuits is called fault analysis. Fault analysis is necessary to improve the customer service reliability and security. Short-circuit currents may change from time to time. Therefore, a suitable fault analysis method is required for calculating the new settings of the protective elements (reclosers, sectionalizer switches, fuses etc.). Also short-circuit calculations are required to determine the short-circuit ratings of new switchgear and substation equipment to be installed in the system. Fault analysis can also be helpful in estimating the size of the additional reactors or fault current limiters which may be required to be inserted in the system to limit the short-circuit currents to a safe value which is below the withstand capacity of the installed circuit-breakers. Usually, the effect of load is neglected during short-circuit analysis [17-21] 
Majority of faults occurring on power systems are The liberalized system of equations is solved to determine unsymmetrical faults, however, the circuit breaker rated the next guess $(m+1)$ of voltage magnitude and angles MVA breaking capacity is based on three-phase base on: symmetrical faults. The main reason is that a three-phase fault produces the greatest fault current and causes the greatest damage to a power system when compare to unsymmetrical faults. The single line-to-ground fault mostly occurring very close to a solidly rounded generator terminal. For proper choice of circuit breakers and protective relaying, we must estimate the magnitude of currents that would flow under short circuit conditions-this is the scope of fault analysis. The three phase balanced fault information is used to select and set phase relays, while line-to-ground fault is used for ground relays. [22-25]

1.1 Need for Evaluation of Fault Analysis in Power System Network:

The fault analysis is one of the basic problems in power system engineering. The results of power system fault analysis are used to determine the type and size of the protective system to be installed on the system so that continuity of supply is ensured even when there is a fault on the power system. The system being planned is to be optimal with respect to construction cost, performance and operating efficiency. For this we can use better planning tools. The major power system tools are load Flow Analysis, short circuit analysis or fault calculations, stability analysis etc. The main purpose of an electrical power system is to generate and supply electrical energy to consumers with reliability and economy. The continuity of power system network is based on the short circuit fault analysis. The evaluation of fault currents on a power system is significant because the protective devices to be installed on the system depend on the values of the fault currents.

\section{PROBLEM FORMULATION OF SYMMETRICAL FAULT ANALYSIS}

\subsection{Preliminary Calculations:}

In cause of fault studies, it is necessary to know the prefault voltages and currents. These pre-fault conditions can be obtained from the results of load flow studies by the Newton Raphson method. The NR method consists high accuracies obtained in a few iterations. The number of iterations remains practically constant irrespective of the size of the power system.NR method begins with initial guesses of all unknown variables (voltage magnitude and angles at Load Buses and voltage angles at Generator Buses). The result is a linear system of equations that can be expressed as:

$$
\left[\begin{array}{l}
\Delta P \\
\Delta Q
\end{array}\right]=\left[\begin{array}{ll}
J 1 & J 2 \\
J 3 & J 3
\end{array}\right]\left[\begin{array}{c}
\Delta \delta \\
\Delta|V|
\end{array}\right]
$$

Where $\Delta P$ and $\Delta Q$ are called the mismatch equations. Real and reactive power in bus is:

$$
\begin{gathered}
P_{i}=\sum_{j=1}^{n}\left|V_{i}\right|\left|V_{j}\right|\left|Y_{i j}\right| \cos \left(\delta_{i}-\delta_{j}-\theta_{i j}\right) \\
Q_{i}=\sum_{j=1}^{n}\left|V_{i}\right|\left|V_{j}\right|\left|Y_{i j}\right| \sin \left(\delta_{i}-\delta_{j}-\theta_{i j}\right)
\end{gathered}
$$

$$
\begin{aligned}
& \delta_{i}^{(k+1)}=\Delta \delta_{i}^{(k)}+\delta_{i}^{k} \\
& \left|V_{i}^{(k+1)}\right|=\Delta V_{i}^{(k)}+V_{i}^{k}
\end{aligned}
$$

Where, $i$ and $j$ are ith and $j$ th buses and $k$ is no. of iterations. The flow chart of NR method is depicted in Figure below:

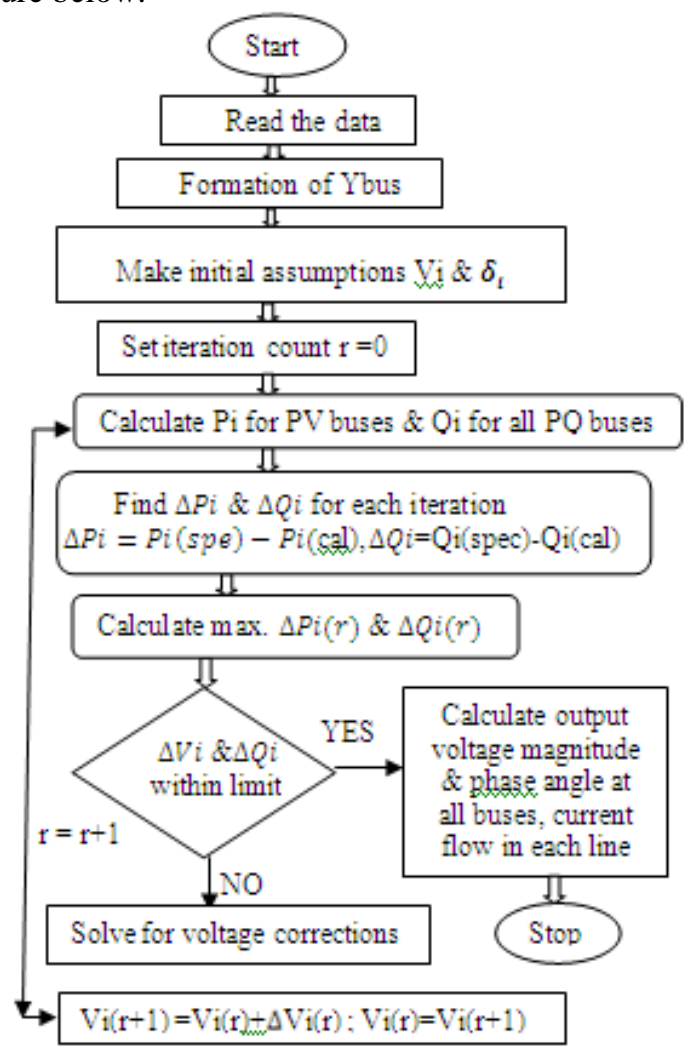

Fig 1: Flow Chart for Newton Raphson Method

\subsection{Symmetrical Fault Analysis:}

In transmission line faults, roughly $5 \%$ are symmetric. It is the most infrequent fault but the most severe type of fault encountered because the network is balanced, it is solved on per-phase basis. A general representation of a balanced three-phase fault is shown in Figure below 2. Where $F$ is the fault point with impedances $\mathrm{Zf}$ and $\mathrm{Zg}$ and $\mathbf{Z}_{\mathbf{1}}$ is positive sequence impedance.

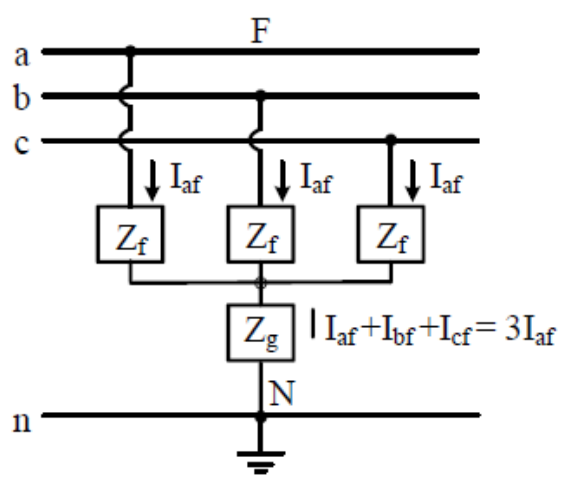

Fig 2: General representation of LLL fault 


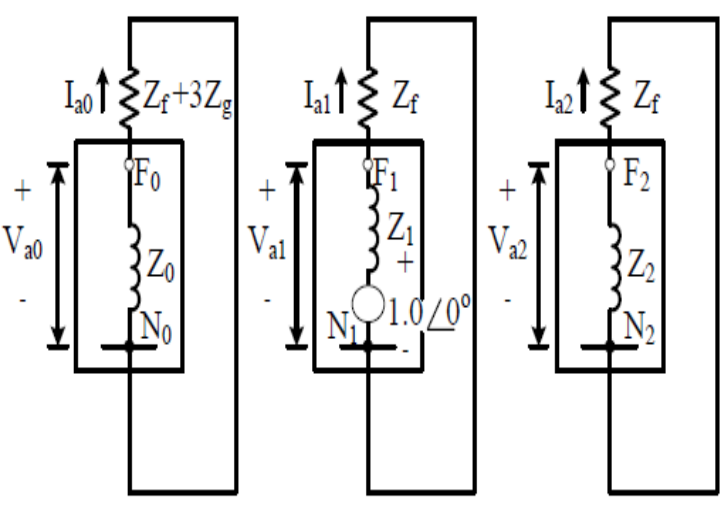

Fig.3: sequence network representation of LLL fault

From Fig. 3 it can be noticed that the only has an internal voltage source is the positive-sequence network. Therefore, the corresponding currents for each of the sequences can be expressed as

$$
\begin{aligned}
& \mathrm{I}_{\mathrm{a} 1}=\mathrm{I}_{\mathrm{a} 2}=0=0 \\
& \mathrm{I}_{\mathrm{a} 1}=\frac{\mathrm{V}_{\mathrm{s}}}{\mathrm{Z}_{1}+\mathrm{Z}_{\mathrm{f}}} \\
& {\left[\begin{array}{c}
I_{a f} \\
I_{b f} \\
I_{c f}
\end{array}\right]=\left[\begin{array}{ccc}
1 & 1 & 1 \\
1 & a^{2} & a \\
1 & a & a^{2}
\end{array}\right]\left[\begin{array}{c}
0 \\
I_{a 1} \\
0
\end{array}\right] }
\end{aligned}
$$

From equation $6,7,8, \& 9$ can solve that,

$$
\begin{aligned}
& \mathrm{I}_{\mathrm{af}}=\mathrm{I}_{\mathrm{a} 1}=\frac{1.0<0^{\circ}}{\mathrm{Z}_{1}+\mathrm{Z}_{\mathrm{f}}} \\
& \text { And also similarly, } \\
& V_{a 0}=V_{a 2}=0 \\
& V_{a 1}=Z_{f} I_{a 1} \\
& {\left[\begin{array}{c}
V_{a f} \\
V_{b f} \\
V_{c f}
\end{array}\right]=\left[\begin{array}{ccc}
1 & 1 & 1 \\
1 & a^{2} & a \\
1 & a & a^{2}
\end{array}\right]\left[\begin{array}{c}
0 \\
V_{a 1} \\
0
\end{array}\right]}
\end{aligned}
$$

Above equations can solve that, $\mathrm{V}_{\mathrm{af}}=\mathrm{Z}_{\mathrm{f}} \mathrm{I}_{\mathrm{a} 1}$

The admittance bus matrix formed and used in load flow analysis has to be inverted to obtain the impedance bus matrix for easy calculation process. It is described in terms of modifying an existing bus impedance matrix designated as $\mid$ Zbus $\mid$ old. This new modified matrix is designated as |Zbus $\mid$ new. The injected bus currents in terms of bus voltages for a n-bus network is calculated as:

$$
\mathrm{I}_{\text {bus }}=\mathrm{Y}_{\text {bus }} \cdot \mathrm{V}_{\text {bus }}
$$

Where $I_{b u s}$ is the bus current vector entering the bus \& $\mathrm{Y}_{\text {bus }}$ is the bus admittance matrix For a fault at bus $\mathrm{k}$, the current entering every bus except the faulted bus $\mathrm{k}$ is zero thus equation (16) becomes:

$\left[\begin{array}{c}0 \\ 0 \\ \vdots \\ -\mathrm{Ik}(\mathrm{F}) \\ 0\end{array}\right]=\left[\begin{array}{cccc}\mathrm{Y} 11 & \ldots & \mathrm{Y} 1 \mathrm{k} & \mathrm{Y} 1 \mathrm{n} \\ \mathrm{Y} 21 & \cdots & \mathrm{Y} 2 \mathrm{k} & \mathrm{Y} 2 \mathrm{n} \\ \vdots & \ddots & & \vdots \\ \mathrm{Yk} 1 & \cdots & \mathrm{Ykk} & \mathrm{Ykn} \\ \mathrm{Yn} 1 & \cdots & \mathrm{Ynk} & \mathrm{Ynn}\end{array}\right]\left[\begin{array}{c}\Delta \mathrm{V} 1 \\ \Delta \mathrm{V} 2 \\ \vdots \\ \Delta \mathrm{Vk} \\ \Delta \mathrm{Vn}\end{array}\right]$

Above eq.can be Wright as

$\mathrm{I}_{\text {bus }}(\mathrm{F})=\mathrm{Y}_{\text {bus }} \cdot \Delta \mathrm{V}_{\text {bus }}$

From above equation can get;

$\Delta$ Vbus $=$ inverse (Ybus).Ibus (F)

$$
\begin{aligned}
& =\mathrm{Z}_{\text {bus }} \cdot \mathrm{I}_{\text {bus }}(\mathrm{F}) \\
& \mathrm{V}_{\text {bus }}=\mathrm{V}_{\text {bus }}(0)+\Delta \mathrm{V}_{\text {bus }} \\
& {\left[\begin{array}{c}
\mathrm{V} 1(\mathrm{~F}) \\
\mathrm{V} 2(\mathrm{~F}) \\
\vdots \\
\mathrm{Vk}(\mathrm{F}) \\
\mathrm{Vn}(\mathrm{F})
\end{array}\right]=\left[\begin{array}{c}
\mathrm{V} 1(0) \\
\mathrm{V} 2(0) \\
\vdots \\
\mathrm{Vk}(0) \\
\mathrm{Vn}(0)
\end{array}\right]+\left[\begin{array}{ccccc}
\mathrm{Z} 11 & \ldots & \mathrm{Z} 1 \mathrm{k} & \mathrm{Z} 1 \mathrm{n} \\
\mathrm{Z} 21 & \ddots & \mathrm{Z} 2 \mathrm{k} & \mathrm{Z} 2 \mathrm{n} \\
\vdots & \ddots & & \vdots & \\
\mathrm{Zk} 1 & \cdots & \mathrm{Zkk} & \mathrm{Zkn} \\
\mathrm{Zn} 1 & & \mathrm{Znk} & \mathrm{Znn}
\end{array}\right]\left[\begin{array}{c}
0 \\
0 \\
\vdots \\
-\mathrm{Ik}(\mathrm{F}) \\
0
\end{array}\right]}
\end{aligned}
$$

From eq. \& can get ;

$\mathrm{V}_{\text {bus }}=\mathrm{V}_{\text {bus }}(0)+\mathrm{Z}_{\text {bus }} \mathrm{I}_{\text {bus }}(\mathrm{F})$

The voltage at bus k during the fault is $\mathrm{Vk}(\mathrm{F})$. So;

$\mathrm{V}_{\mathrm{k}}(\mathrm{F})=\mathrm{V}_{\mathrm{k}}(0)-\mathrm{Z}_{\mathrm{kk}} \mathrm{I}_{\mathrm{k}}(\mathrm{F})$

But $\mathrm{V}_{\mathrm{k}}(\mathrm{F})=\mathrm{Z}^{\mathrm{f}} \mathrm{I}_{\mathrm{k}}(\mathrm{F})$, equation (23) becomes;

$\mathrm{Z}^{\mathrm{f}} \mathrm{I}_{\mathrm{k}}(\mathrm{F})=\mathrm{V}_{\mathrm{k}}(0)-\mathrm{V}_{\mathrm{k}}(\mathrm{F})$

Solving $I_{k}(F)$ for gives;

$I_{k}(F)=\frac{V_{k}(0)}{Z_{K K}+Z_{f}}$

Where, $Z_{\mathrm{kk}}=Z_{1}$; for symmetrical faults (LLL Fault) For any bus $i$ the bus voltage during fault is;

$\mathrm{V}_{\mathrm{i}}(\mathrm{F})=\mathrm{V}_{\mathrm{i}}(0)-\mathrm{Z}_{\mathrm{ik}} \mathrm{I}_{\mathrm{k}}(\mathrm{F})$

The short circuit current in the line connected between bus $i$ and $j$ is; $\quad I_{i j}(F)=\frac{V_{i}(F)-V_{j}(F)}{Z_{i j}}$

\subsection{Selection of Rating of Circuit Breakers:}

The use of circuit breaker under short circuit conditions are open the contacts to clear the fault, to close the contacts onto a fault and to carry fault current for a short time while another circuit breaker is clearing the fault. The Breaking capacity of a circuit breaker is of two types i.e. Symmetrical Breaking capacity and Asymmetrical Breaking capacity. Symmetrical short circuit current is obtained by using sub transient reactance for synchronous machines. Momentary current (rms) is then calculated by multiplying the symmetrical momentary current by a factor of 1.6 to account for the presence of dc offset current. The current that a circuit breaker can interrupt is inversely proportional to the operating voltage over a certain range of time. Obviously, rated MVA interrupting capacity of a circuit breaker is to be more than (or equal to) to the short circuit MVA required to be interrupted. If voltage $\&$ current in p.u. values on a 3 ph. Basis, then

$\operatorname{SCMVA}(3 \mathrm{ph})=\mid$ Vprefault $|*| \mathrm{Isc} \mid *($ MVA base $)$

\section{IMPLEMENTATION PROCEDURE FOR SYMMETRICAL FAULT (LLL)}

Algorithm for symmetrical fault analysis for power system network is below:

STEP 1: Read the bus data, line data and load data.

STEP 2: Run the Load flow with N-R method.

STEP 3: Obtain pre-fault voltages at all buses and Currents in all lines using load flow study.

STEP 4: Find Bus impedance matrix by inverting the bus Admittance matrix or using builds Programming $\left(\mathrm{Z}_{\text {bus }}=\mathrm{Z}\right.$ build $\left(\mathrm{Z}_{1}\right.$ data $)$

STEP 5: Choose MVAbase, KVbase \& calculate Ibase.

STEP 6: Specify the faulty bus \& obtain current at the Faulty bus and bus voltages during fault at all Buses using Eq. (23) and (24) 
STEP 7: Find current flows in each line of the power System network using equation (26).

STEP 8: Calculate SCMVA rating of circuit breaker (Choose acc. to the fault current magnitude) for Each transmission Line and each buses of IEEE 6 bus and 30 bus system using equation (27)

The flow chart of symmetrical fault on IEEE bus system is show in below figure

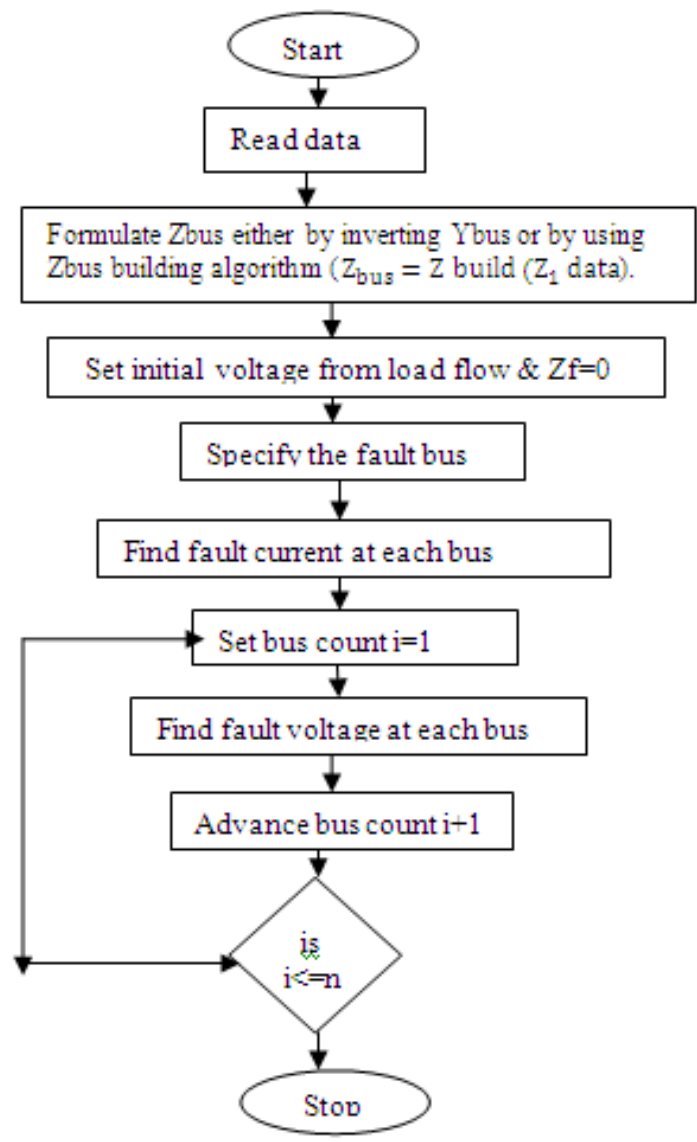

Fig 4: Flow Chart for the Calculations of Symmetrical Fault

\section{RESULTS AND DISCUSSION}

\subsection{Symmetrical fault (LLL) on IEEE 6 bus system:}

Firstly, discuss the results when symmetrical fault (LLL Fault) occurs on IEEE 6 bus system. Pre-fault voltages are carried out by using NR method. After the pre-fault calculations, a LLL fault was simulated on the 6 bus system then calculated the fault voltage magnitude at each bus, fault current flows in the lines, SCMVA ratings based on the fault currents on each bus and lines was also calculated then the corresponding Circuit Breakers ratings are choose.

- Table 1: Prefault Voltage magnitude, phase Angle, Real and Reactive Powers using NR Method on IEEE 6 bus system :

\begin{tabular}{|l|l|l|l|l|l|l|}
\hline $\begin{array}{l}\text { Bus } \\
\text { no }\end{array}$ & $\begin{array}{l}\text { Voltage } \\
\text { magnitu } \\
\text { de (p.u) }\end{array}$ & $\begin{array}{l}\text { Angle } \\
\text { (degree) }\end{array}$ & $\begin{array}{l}\text { MW } \\
(\mathbf{L})\end{array}$ & $\begin{array}{l}\text { Mvar } \\
(\mathbf{L})\end{array}$ & $\begin{array}{l}\text { MW } \\
(\mathbf{G})\end{array}$ & $\begin{array}{l}\text { Mvar } \\
(\mathbf{G})\end{array}$ \\
\hline 1 & 1.06 & 0.000 & 0.00 & 0.00 & 649.7 & 231.7 \\
\hline 2 & 0.99 & -30.448 & 150.0 & 0.00 & 0.00 & 174.4 \\
\hline 3 & 1.00 & -17.396 & 100.0 & 0.00 & 0.00 & 85.9 \\
\hline 4 & 0.93 & -27.178 & 100.0 & 70.00 & 0.00 & 0.00 \\
\hline 5 & 0.968 & -14.901 & 90.00 & 30.0 & 0.00 & 0.00 \\
\hline 6 & 0.868 & -21.177 & 160.0 & 110.0 & 0.00 & 0.00 \\
\hline $\begin{array}{l}\text { Tota } \\
1\end{array}$ & & & 600.0 & 210.0 & 649.7 & 492.0 \\
\hline
\end{tabular}

- Table 2: Compare the pre-fault voltage magnitude in p.u. and fault voltage magnitude in p.u at each bus:

\begin{tabular}{|l|l|l|l|l|l|l|l|}
\hline \multirow{2}{*}{$\begin{array}{l}\text { Bus } \\
\text { no }\end{array}$} & $\begin{array}{l}\text { Pre-fault } \\
\text { voltage } \\
\text { magnitud } \\
\text { e (p.u) }\end{array}$ & \multicolumn{6}{|c|}{ When Fault at bus no. } \\
\cline { 3 - 8 } & & $\mathbf{1}$ & $\mathbf{2}$ & $\mathbf{3}$ & $\mathbf{4}$ & $\mathbf{5}$ & $\mathbf{6}$ \\
\hline 1 & 1.06 & 0.00 & 0.64 & 0.58 & 0.55 & 0.44 & 0.50 \\
\hline 2 & 0.99 & 0.61 & 0.00 & 0.76 & 0.19 & 0.70 & 0.51 \\
\hline 3 & 1.00 & 0.43 & 0.71 & 0.00 & 0.64 & 0.14 & 0.53 \\
\hline 4 & 0.93 & 0.48 & 0.17 & 0.66 & 0.00 & 0.59 & 0.43 \\
\hline 5 & 0.968 & 0.32 & 0.64 & 0.22 & 0.56 & 0.00 & 0.43 \\
\hline 6 & 0.868 & 0.38 & 0.43 & 0.50 & 0.33 & 0.39 & 0.00 \\
\hline
\end{tabular}

Table 2 represents the Comparison between the pre-fault voltage magnitude in p.u. and fault voltage magnitude at each bus and it is observed that when a LLL fault occurs, the voltage at faulted bus is reduced to zero and voltage magnitude on other buses are also affected. Graphical Representation of post fault voltage magnitude in p.u when fault occurs at different buses of IEEE 6 bus system is show in below figure. From below graph it can observed that when fault at bus 1 corresponding voltage at bus no. 1 is zero. And also when fault at bus 2, corresponding voltage at bus 1 is less affected. And also when fault at bus no. 5 , voltage at bus no. 1 is more affected.

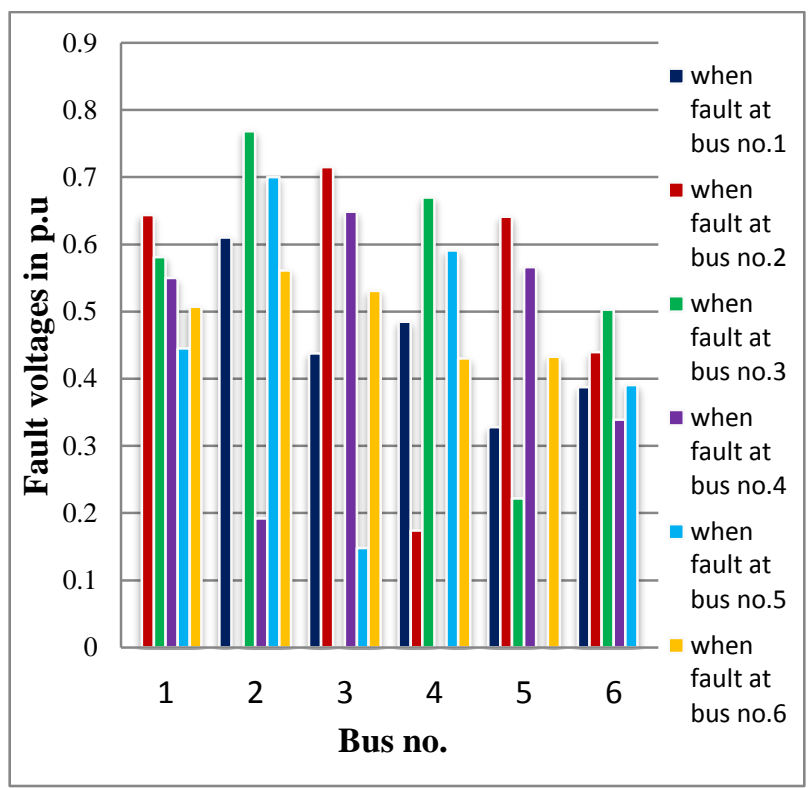

Fig 5: Graphical Representation of post fault voltages when fault occur at

Different buses of IEEE 6 Bus system during LLL fault 
- Table 3: Fault current magnitude, Short circuit MVA and Circuit breaker ratings at each bus:

\begin{tabular}{|l|l|l|l|}
\hline $\begin{array}{l}\text { Bus } \\
\text { no }\end{array}$ & $\begin{array}{l}\text { Fault current } \\
\text { magnitude(pu) }\end{array}$ & $\begin{array}{l}\text { SCMVA } \\
\text { (MVA) }\end{array}$ & $\begin{array}{l}\text { Circuit Breaker } \\
\text { Rating (MVA) }\end{array}$ \\
\hline 1 & 12.8156 & 1358 & 1360 \\
\hline 2 & 12.0488 & 1192 & 1200 \\
\hline 3 & 9.6018 & 960.18 & 970 \\
\hline 4 & 11.0808 & 1030.5 & 1040 \\
\hline 5 & 10.1313 & 980.70 & 990 \\
\hline 6 & 8.9825 & 780.5 & 790 \\
\hline
\end{tabular}

From Table 3 can observed that bus no. 1 has higher fault current and circuit breaker rating i.e 12.8156 p.u, 1360 MVA.

- Table 4 : Comparison of Pre \& post Fault Currents in Each Line of IEEE 6 bus system during Symmetrical fault (LLL) :

\begin{tabular}{|l|l|l|l|l|l|l|l|}
\hline $\begin{array}{l}\text { Line } \\
\text { no. }\end{array}$ & $\begin{array}{l}\text { Pre-fault } \\
\text { Current } \\
\text { (p.u) }\end{array}$ & \multicolumn{7}{|c|}{ When fault at bus no. } \\
\cline { 3 - 8 } & 0.0296 & $\begin{array}{l}2.1 \\
3\end{array}$ & $\begin{array}{l}2.0 \\
8\end{array}$ & 1.27 & $\underline{\mathbf{2 . 4 2}}$ & 1.06 & 0.92 \\
\hline 1 & & $\begin{array}{l}3.7 \\
2\end{array}$ & $\begin{array}{l}1.3 \\
8\end{array}$ & 3.33 & 1.08 & $\underline{\mathbf{4 . 1 3}}$ & 1.03 \\
\hline 2 & 0.0102 & $\begin{array}{l}1.6 \\
3\end{array}$ & $\begin{array}{l}1.1 \\
2\end{array}$ & 0.73 & 1.07 & 0.42 & $\underline{\mathbf{2 . 3 1}}$ \\
\hline 3 & 0.02073 & $\begin{array}{l}3.7 \\
2\end{array}$ & $\begin{array}{l}4.9 \\
8\end{array}$ & 3.11 & $\underline{\mathbf{5 . 4 8}}$ & 3.29 & 3.77 \\
\hline 4 & 0.0021 & $\begin{array}{l}2.6 \\
7\end{array}$ & $\begin{array}{l}1.9 \\
5\end{array}$ & $\underline{\mathbf{5 . 2 8}}$ & 2.06 & 3.52 & 2.35 \\
\hline 5 & 0.00147 & $\begin{array}{l}1.2 \\
3\end{array}$ & $\begin{array}{l}2.0 \\
7\end{array}$ & 1.55 & 2.64 & 1.73 & $\underline{\mathbf{3 . 3 6}}$ \\
\hline 6 & 0.00794 & $\begin{array}{l}0.1 \\
7\end{array}$ & $\begin{array}{l}1.1 \\
4\end{array}$ & 1.64 & 1.28 & 2.20 & $\underline{\mathbf{2 . 4 4}}$ \\
\hline 7 & 0.01710 & & & & & & \\
\hline
\end{tabular}

From the Table 4 , it is analysed that in line no. 1 maximum fault current flowing up to $2.42 \mathrm{pu}$. when there is a fault at bus no. 4 \& line no. 1 is most effected when there is a fault at bus no. 5 \& line no. 2 is most effected similarly check other lines and this effect is clearly shown from graphical representation of current flow in each line when short circuit fault occurs at different buses as shown in Fig.6 Now Choose the SCMVA rating of Circuit Breaker for each line according to the Table 4.

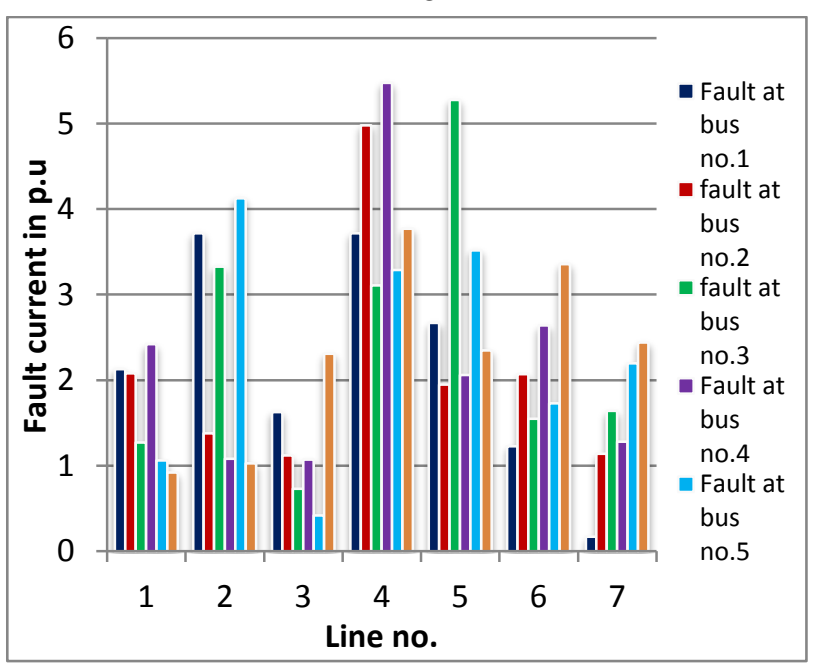

Figure 6: Graphical Representation of transmission line currents when fault occur at different buses
Table 5 :SCMVA \& Circuit Breaker Ratings in each line :

\begin{tabular}{|l|l|l|}
\hline $\begin{array}{c}\text { Line } \\
\text { no. }\end{array}$ & SCMVA rating & $\begin{array}{c}\text { Circuit Breaker rating } \\
\text { (MVA) }\end{array}$ \\
\hline 1 & 225.45 & 230 \\
\hline 2 & 399.89 & 400 \\
\hline 3 & 201.36 & 210 \\
\hline 4 & 510.14 & 520 \\
\hline 5 & 528.85 & 530 \\
\hline 6 & 291.65 & 300 \\
\hline 7 & 211.83 & 220 \\
\hline
\end{tabular}

4.2 Symmetrical fault (LLL) on IEEE 30 bus system:

Load flow analysis was carried out using the NR load flow method then after the pre-fault calculations, LLL fault was simulated on Each Bus of the IEEE 30 bus system then calculated the fault voltage magnitude at each bus, fault currents flows in the lines, SCMVA ratings based on the fault currents on each bus and line then the corresponding Circuit Breakers ratings are choosed.

- $\quad$ Table 6 : Voltage magnitude, phase Angle, Real $\&$ Reactive Powers by using NR Method on IEEE 30 bus system :

\begin{tabular}{|c|c|c|c|c|c|c|}
\hline $\begin{array}{l}\text { Bus } \\
\text { no }\end{array}$ & $\begin{array}{l}\text { Prefaul } \\
\text { t } \\
\text { voltage } \\
\text { (p.u) }\end{array}$ & $\begin{array}{l}\text { Angle } \\
\text { (degree } \\
\text { ) }\end{array}$ & $\begin{array}{l}\text { MW } \\
\text { (L) }\end{array}$ & $\begin{array}{l}\text { Mva } \\
\mathbf{r} \\
(\mathbf{L})\end{array}$ & $\begin{array}{l}\text { MW } \\
\text { (G) }\end{array}$ & $\begin{array}{l}\text { Mva } \\
\mathbf{r} \\
\text { (G) }\end{array}$ \\
\hline 1 & 1.06 & 0.000 & 0.00 & 0.000 & 172.3 & -1.43 \\
\hline 2 & 1.04 & -3.534 & 21.7 & 12.70 & 40.00 & 30.05 \\
\hline 3 & 1.02 & -5.174 & 2.40 & 1.200 & 0.000 & 0.0 \\
\hline 4 & 1.01 & -6.209 & 7.60 & 1.600 & 0.000 & 0.0 \\
\hline 5 & 1.01 & -10.125 & 94.2 & 19.00 & 20.00 & 26.24 \\
\hline 6 & 1.016 & -7.318 & 0.00 & 0.00 & 0.00 & 0.00 \\
\hline 7 & 1.006 & -8.996 & 22.8 & 10.90 & 0.00 & 0.00 \\
\hline 8 & 1.01 & -7.52 & 30.0 & 30.60 & 20.00 & 14.83 \\
\hline 9 & 1.053 & -8.65 & 0.00 & 0.000 & 0.000 & 0.0 \\
\hline 10 & 1.047 & -10.49 & 5.80 & 2.000 & 0.000 & 0.0 \\
\hline 11 & 1.082 & -6.564 & 0.00 & 0.000 & 20.00 & 15.18 \\
\hline 12 & 1.06 & -9.55 & 11.2 & 7.500 & 0.000 & 0.00 \\
\hline 13 & 1.071 & -8.13 & 0.00 & 0.000 & 20.00 & 7.814 \\
\hline 14 & 1.047 & -10.477 & 6.20 & 1.600 & 0.000 & 0.00 \\
\hline 15 & 1.042 & -10.606 & 8.20 & 2.500 & 0.000 & 0.0 \\
\hline 16 & 1.031 & -10.221 & 3.50 & 1.800 & 0.000 & 0.0 \\
\hline 17 & 1.028 & -10.621 & 9.00 & 5.800 & 0.000 & 0.0 \\
\hline 18 & 1.032 & -11.259 & 3.20 & 0.900 & 0.000 & 0.0 \\
\hline 19 & 1.032 & -11.456 & 9.50 & 3.400 & 0.000 & 0.0 \\
\hline 20 & 1.038 & -11.274 & 2.20 & 0.700 & 0.000 & 0.0 \\
\hline 21 & 1.032 & -10.968 & 17.5 & 11.20 & 0.000 & 0.0 \\
\hline 22 & 1.026 & -10.963 & 0.00 & 0.000 & 0.00 & 0.0 \\
\hline 23 & 1.022 & -11.114 & 3.20 & 1.600 & 0.000 & 0.0 \\
\hline 24 & 1.004 & -11.452 & 8.70 & 6.700 & 0.000 & 0.0 \\
\hline 25 & 1.027 & -11.475 & 0.00 & 0.000 & 0.000 & 0.0 \\
\hline 26 & 1.014 & -11.892 & 3.50 & 2.300 & 0.000 & 0.0 \\
\hline 27 & 1.016 & -11.231 & 0.00 & 0.00 & 0.000 & 0.0 \\
\hline 28 & 1.014 & -7.805 & 0.00 & 0.000 & 0.000 & 0.0 \\
\hline 29 & 1.008 & -12.452 & 2.40 & 0.900 & 0.00 & 0.0 \\
\hline 30 & 0.996 & -13.329 & 10.6 & 1.900 & 0.000 & 0.0 \\
\hline Total & & & 283.4 & 126.8 & 292.3 & 92.70 \\
\hline
\end{tabular}


- $\quad$ Table 7: fault current \&circuit breaker ratings for each bus

\begin{tabular}{|l|l|l|l|}
\hline $\begin{array}{l}\text { Bus } \\
\text { no. }\end{array}$ & $\begin{array}{l}\text { Fault Current } \\
\text { (pu) }\end{array}$ & $\begin{array}{l}\text { SCMVA } \\
\text { Rating }\end{array}$ & $\begin{array}{l}\text { Circuit Barker } \\
\text { Rating (MVA) }\end{array}$ \\
\hline 1 & 22.5228 & 2387.102 & 2400 \\
\hline 2 & 25.510 & 2653.03 & 2660 \\
\hline 3 & 18.329 & 1882.026 & 1900 \\
\hline 4 & 24.198 & 2465.5 & 2470 \\
\hline 5 & 13.929 & 14060.4 & 1470 \\
\hline 6 & 23.04 & 2442.21 & 2450 \\
\hline 7 & 11.719 & 1178.89 & 1180 \\
\hline 8 & 12.645 & 1277.01 & 1280 \\
\hline 9 & 6.361 & 670.12 & 680 \\
\hline 10 & 6.259 & 650.6 & 660 \\
\hline 11 & 2.901 & 313.25 & 320 \\
\hline 12 & 5.678 & 611.3 & 620 \\
\hline 13 & 3.291 & 352.6 & 360 \\
\hline 14 & 3.250 & 348.34 & 350 \\
\hline 15 & 4.563 & 474.5 & 480 \\
\hline 16 & 4.025 & 421.82 & 430 \\
\hline 17 & 4.779 & 486.65 & 490 \\
\hline 18 & 3.260 & 364.132 & 370 \\
\hline 19 & 3.307 & 373.18 & 380 \\
\hline 20 & 3.528 & 386.120 & 390 \\
\hline 21 & 5.0821 & 546.32 & 550 \\
\hline 22 & 5.043 & 540.85 & 550 \\
\hline 23 & 3.3065 & 364.87 & 370 \\
\hline 24 & 3.9018 & 417.89 & 420 \\
\hline 25 & 2.7726 & 282.74 & 290 \\
\hline 26 & 1.2543 & 125.53 & 130 \\
\hline 27 & 3.0521 & 313.44 & 320 \\
\hline 28 & 11.1029 & 1125.83 & 1130 \\
\hline 29 & 1.5446 & 155.48 & 160 \\
\hline 30 & 1.4154 & 140.832 & 150 \\
\hline & & & \\
\hline
\end{tabular}

- Table 8:Compare the pre-fault voltage magnitude in p.u. and fault voltage magnitude in p.u for IEEE 30 Bus system:

\begin{tabular}{|c|c|c|c|c|c|c|c|c|c|c|c|c|c|c|c|c|c|}
\hline \multirow{2}{*}{$\begin{array}{l}\text { Bus } \\
\text { no }\end{array}$} & \multirow{2}{*}{$\begin{array}{l}\text { Pre } \\
\text { Fault } \\
\text { voltage } \\
\text { (p.u) }\end{array}$} & \multicolumn{16}{|c|}{ When fault at bus no. } \\
\hline & & $\mathbf{1}$ & 3 & 4 & 5 & 6 & 9 & 10 & 14 & 16 & 17 & 23 & 24 & 26 & 27 & 28 & 30 \\
\hline 1 & 1.06 & 0.00 & 0.73 & 0.70 & 0.90 & 0.76 & 0.97 & 0.98 & 1.01 & 1.00 & 0.99 & 1.01 & 1.01 & 1.04 & 1.02 & 0.92 & 1.04 \\
\hline 2 & $\begin{array}{l}1.04 \\
\end{array}$ & 0.51 & 0.75 & 0.63 & 0.79 & 0.64 & 0.93 & 0.94 & 0.99 & 0.97 & 0.96 & 0.99 & 0.98 & 1.02 & 0.99 & 0.85 & 1.02 \\
\hline 3 & 1.02 & 0.64 & 0.00 & 0.22 & 0.86 & 0.50 & 0.87 & 0.87 & 0.94 & 0.92 & 0.90 & 0.94 & 0.93 & 0.99 & 0.95 & 0.77 & 0.99 \\
\hline 4 & 1.01 & 0.71 & 0.42 & 0.00 & 0.83 & 0.37 & 0.83 & 0.83 & 0.91 & 0.88 & 0.87 & 0.91 & 0.90 & 0.98 & 0.93 & 0.70 & 0.97 \\
\hline 5 & 1.01 & 0.77 & 0.80 & 0.69 & 0.00 & 0.60 & 0.90 & 0.91 & 0.96 & 0.95 & 0.93 & 0.96 & 0.95 & 0.99 & 0.95 & 0.81 & 0.98 \\
\hline 6 & 1.016 & 0.74 & 0.61 & 0.33 & 0.76 & 0.00 & 0.76 & 0.77 & 0.91 & 0.87 & 0.83 & 0.90 & 0.87 & 0.96 & 0.89 & 0.53 & 0.95 \\
\hline 7 & 1.006 & 0.75 & 0.68 & 0.48 & 0.45 & 0.25 & 0.82 & 0.82 & 0.92 & 0.90 & 0.87 & 0.91 & 0.89 & 0.96 & 0.91 & 0.64 & 0.96 \\
\hline 8 & 1.01 & 0.74 & 0.61 & 0.34 & 0.76 & 0.16 & 0.73 & 0.77 & 0.90 & 0.87 & 0.83 & 0.89 & 0.86 & 0.95 & 0.87 & 0.44 & 0.94 \\
\hline 9 & 1.053 & 0.77 & 0.60 & 0.30 & 080 & 0.09 & 0.00 & 0.27 & 0.81 & 0.68 & 0.50 & 0.76 & 0.67 & 0.96 & 0.91 & 0.55 & 0.96 \\
\hline 10 & 1.047 & 0.76 & 0.58 & 0.28 & 0.81 & 0.14 & 0.28 & 0.00 & 0.74 & 0.55 & 0.31 & 0.67 & 0.55 & 0.93 & 0.87 & 0.55 & 0.94 \\
\hline 11 & 1.082 & 0.79 & 0.62 & 0.31 & 0.83 & 0.09 & 0.00 & 0.28 & 0.83 & 0.69 & 0.52 & 0.78 & 0.69 & 0.98 & 0.87 & 0.56 & 0.99 \\
\hline 12 & 1.06 & 0.76 & 0.52 & 0.18 & 0.84 & 0.26 & 0.52 & 0.47 & 0.51 & 0.47 & 0.53 & 0.61 & 0.66 & 0.96 & 0.87 & 0.63 & 0.97 \\
\hline 13 & 1.071 & 0.76 & 0.53 & 0.18 & 0.85 & 0.26 & 0.59 & 0.47 & 0.51 & 0.47 & 0.54 & 0.62 & 0.67 & 0.97 & 0.88 & 0.64 & 0.98 \\
\hline 14 & 1.047 & 0.75 & 0.53 & 0.21 & 0.83 & 0.25 & 0.55 & 0.42 & 0.00 & 0.48 & 0.51 & 0.52 & 0.60 & 0.94 & 0.84 & 0.61 & 0.95 \\
\hline 15 & 1.042 & 0.75 & 0.54 & 0.22 & 0.82 & 0.23 & 0.52 & 0.37 & 0.40 & 0.50 & 0.49 & 0.42 & 0.54 & 0.92 & 0.81 & 0.60 & 0.94 \\
\hline 16 & 1.031 & 0.75 & 0.55 & 0.22 & 0.82 & 0.21 & 0.45 & 0.27 & 0.60 & 0.00 & 0.26 & 0.63 & 0.61 & 0.94 & 0.84 & 0.59 & 0.95 \\
\hline 17 & 1.028 & 0.75 & 0.57 & 0.26 & 0.81 & 0.61 & 0.33 & 0.08 & 0.69 & 0.38 & 0.00 & 0.66 & 0.57 & 0.93 & 0.82 & 0.64 & 0.94 \\
\hline 18 & 1.032 & 0.75 & 0.55 & 0.25 & 0.81 & 0.21 & 0.44 & 0.25 & 0.53 & 0.51 & 0.43 & 0.51 & 0.54 & 0.91 & 0.81 & 0.56 & 0.93 \\
\hline 19 & 1.032 & 0.75 & 0.56 & 0.26 & 0.80 & 0.19 & 0.39 & 0.17 & 0.60 & 0.52 & 0.39 & 0.56 & 0.54 & 0.91 & 0.80 & 0.58 & 0.92 \\
\hline 20 & 1.038 & 0.75 & 0.57 & 0.26 & 0.80 & 0.16 & 0.36 & 0.13 & 0.63 & 0.53 & 0.37 & 0.58 & 0.54 & 0.92 & 0.81 & 0.57 & 0.93 \\
\hline 21 & 1.032 & 0.77 & 0.58 & 0.28 & 0.80 & 0.15 & 0.32 & 0.05 & 0.72 & 0.56 & 0.34 & 0.62 & 0.45 & 0.90 & 0.77 & 0.56 & 0.92 \\
\hline 22 & 1.026 & 0.75 & 0.55 & 0.28 & 0.80 & 0.15 & 0.32 & 0.06 & 0.72 & 0.56 & 0.34 & 0.60 & 0.42 & 0.89 & 0.76 & 0.53 & 0.91 \\
\hline 23 & 1.022 & 0.75 & 0.57 & 0.25 & 0.80 & 0.21 & 0.48 & 0.32 & 0.53 & 0.53 & 0.47 & 0.0 & 0.30 & 0.87 & 0.73 & 0.53 & 0.89 \\
\hline 24 & 1.004 & 0.75 & 0.59 & 0.28 & 0.80 & 0.17 & 0.43 & 0.24 & 0.67 & 0.58 & 0.45 & 0.48 & 0.00 & 0.80 & 0.62 & 0.55 & 0.84 \\
\hline 25 & 1.027 & 0.75 & 0.58 & 0.30 & 0.79 & 0.13 & 0.55 & 0.44 & 0.76 & 0.70 & 0.60 & 0.59 & 0.32 & 0.56 & 0.24 & 0.48 & 0.66 \\
\hline 26 & 1.014 & 0.74 & 0.58 & 0.31 & 0.78 & 0.15 & 0.55 & 0.44 & 0.76 & 0.69 & 0.59 & 0.59 & 0.33 & 0.00 & 0.25 & 0.32 & 0.60 \\
\hline 27 & 1.016 & 0.75 & 0.60 & 0.32 & 0.77 & 0.11 & 0.63 & 0.57 & 0.82 & 0.77 & 0.69 & 0.71 & 0.52 & 0.71 & 0.00 & 0.22 & 0.51 \\
\hline 28 & 1.014 & 0.74 & 0.60 & 0.33 & 0.78 & 0.01 & 0.75 & 0.75 & 0.89 & 0.86 & 0.81 & 0.87 & 0.82 & 0.93 & 0.79 & 0.00 & 0.91 \\
\hline 29 & 1.008 & 0.74 & 0.59 & 0.33 & 0.76 & 0.13 & 0.63 & 0.57 & 0.81 & 0.76 & 0.68 & 0.70 & 0.52 & 0.70 & 0.02 & 0.24 & 0.29 \\
\hline 30 & 0.996 & 0.73 & 0.56 & 0.33 & 0.76 & 0.15 & 0.63 & 0.56 & 0.80 & 0.75 & 0.68 & 0.69 & 0.52 & 0.70 & 0.04 & 0.25 & 0.00 \\
\hline
\end{tabular}




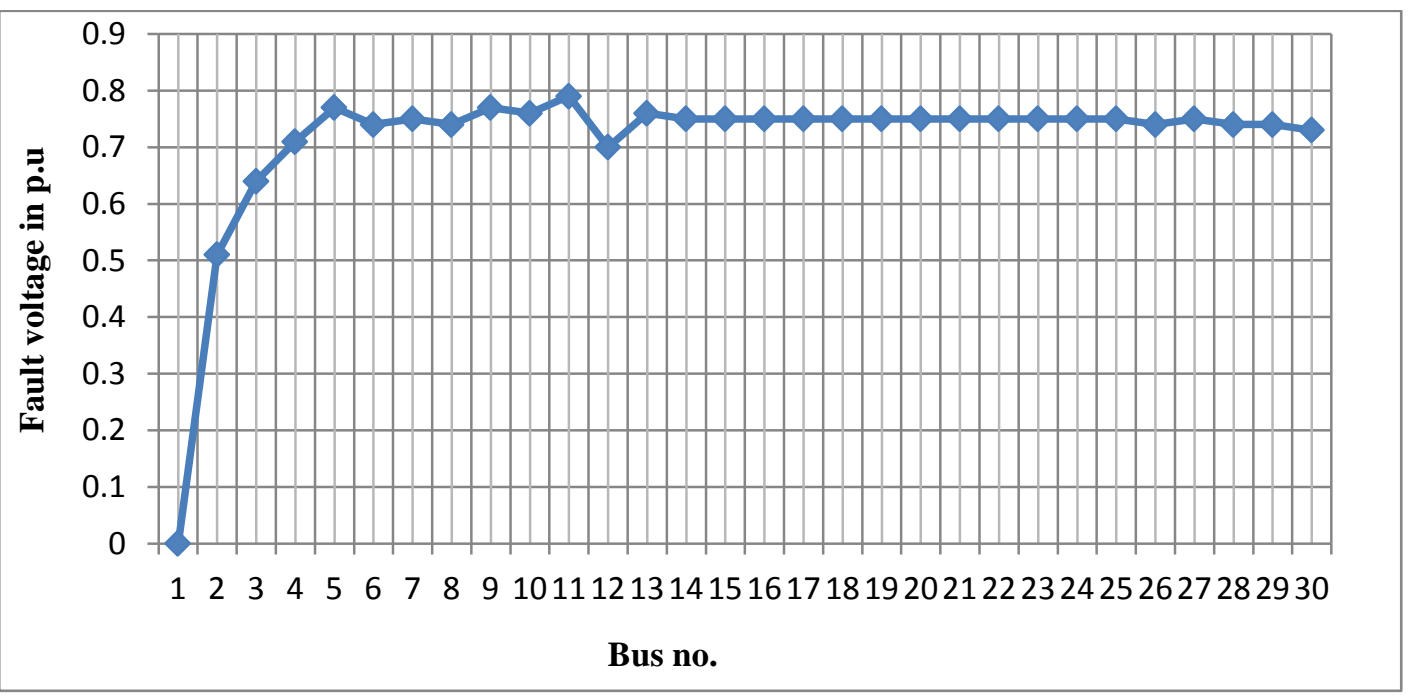

Fig.7: Graphical Representation for fault voltage of IEEE 30 Bus system LLL fault occurs at bus no.1

\begin{tabular}{|c|c|c|c|c|c|c|c|c|c|c|c|c|c|c|c|c|c|}
\hline \multirow{2}{*}{$\begin{array}{l}\text { Lin } \\
\text { e } \\
\text { no }\end{array}$} & \multirow{2}{*}{$\begin{array}{l}\text { From } \\
- \\
\text { To }\end{array}$} & \multirow{2}{*}{$\begin{array}{l}\text { Prefa } \\
\text { ult } \\
\text { curre } \\
\text { nt } \\
\text { (p.u) }\end{array}$} & \multicolumn{15}{|c|}{ When fault occurs at bus no. } \\
\hline & & & 1 & 3 & 4 & 5 & 6 & 8 & 9 & 14 & 16 & 17 & 24 & 26 & 27 & 28 & 30 \\
\hline 1 & $1-2$ & 0.0036 & $\underline{8.45}$ & 0.68 & 1.43 & 1.96 & 2.09 & 1.50 & 1.1 & 1.1 & 1.12 & 1.13 & 1.14 & 1.12 & 1.11 & 1.42 & 1.12 \\
\hline 2 & $1-3$ & 0.0178 & $\overline{\underline{3.76}}$ & 4.27 & 2.85 & 0.51 & 1.58 & 1.04 & 0.7 & 0.7 & 0.71 & 0.72 & 0.71 & 0.62 & 0.64 & 0.97 & 0.61 \\
\hline 3 & $2-4$ & 0.0127 & $\overline{1.10}$ & 1.82 & 3.45 & 0.30 & 1.52 & 1.91 & 0.5 & 0.5 & 0.54 & 0.55 & 0.50 & 0.34 & 0.40 & 0.83 & 0.34 \\
\hline 4 & $3-4$ & 0.0009 & 1.75 & 10.4 & 5.63 & 0.96 & $\begin{array}{l}3.4 \\
\end{array}$ & 1.95 & 1.0 & 0.8 & 0.91 & 0.97 & 0.89 & 0.59 & 0.71 & 1.76 & 0.59 \\
\hline 5 & $2-5$ & 0.0134 & 1.30 & 0.44 & 0.40 & $\underline{3.90}$ & 0.38 & 0.47 & 0.5 & 0.5 & 0.59 & 0.54 & 0.55 & 0.58 & 0.56 & 0.48 & 0.58 \\
\hline 6 & $2-6$ & 0.0168 & 1.26 & 0.79 & 1.58 & 0.35 & 3.49 & 1.95 & 0.9 & 0.5 & 0.62 & 0.72 & 0.67 & 0.47 & 0.60 & 1.76 & 0.48 \\
\hline 7 & $4-6$ & 0.0009 & 0.81 & 4.38 & 7.84 & 1.65 & $\overline{8.62}$ & 4.72 & 1.5 & 0.4 & 0.45 & 0.77 & 0.78 & 0.57 & 0.93 & 4.09 & 0.62 \\
\hline 8 & $5-7$ & 0.0034 & 0.19 & 0.96 & 1.69 & $\underline{3.62}$ & 2.82 & 1.55 & 0.7 & 0.3 & 0.39 & 0.49 & 0.45 & 0.21 & 0.41 & 1.37 & 0.24 \\
\hline 9 & $6-7$ & 0.0005 & 0.18 & 0.90 & 1.69 & $\underline{3.60}$ & 2.90 & 1.52 & 0.7 & 0.1 & 0.14 & 0.13 & 0.39 & 0.30 & 0.40 & 1.32 & 0.31 \\
\hline 10 & 6-8 & 0.0006 & 0.03 & 0.05 & 0.18 & 0.34 & 0.38 & 10.6 & 0.1 & 0.3 & 0.99 & 0.54 & 0.24 & 0.24 & 0.46 & 2.00 & 0.29 \\
\hline 11 & 6-9 & 0.0126 & 0.11 & 0.04 & 0.22 & 0.19 & 0.46 & $\overline{0.36}$ & $\underline{3.6}$ & 0.3 & 0.61 & 1.61 & 1.02 & 0.20 & 0.26 & 0.11 & 0.18 \\
\hline 12 & $6-10$ & 0.0531 & 0.04 & 0.04 & 0.13 & 0.85 & 0.26 & 0.12 & 0.8 & 0.8 & 0.43 & $\underline{0.96}$ & 0.62 & 0.16 & 0.19 & 0.12 & 0.13 \\
\hline 13 & $9-11$ & 0.0094 & 0.16 & 0.11 & 0.06 & 0.17 & 0.02 & 0.11 & 0.0 & 1.6 & 1.21 & 1.79 & 0.14 & 0.21 & 0.18 & 0.04 & 0.21 \\
\hline 14 & $9-10$ & 0.0044 & 0.16 & 0.17 & 0.25 & 0.16 & 0.45 & 0.01 & $\underline{2.5}$ & 0.1 & 1.70 & $\overline{1.39}$ & 1.24 & 0.45 & 0.50 & 0.28 & 0.32 \\
\hline 15 & $4-12$ & 0.0269 & 0.19 & 0.43 & 0.73 & 0.05 & 0.46 & 0.20 & $\overline{0.9}$ & 1.8 & 0.14 & 0.01 & 1.07 & 0.33 & 0.38 & 0.12 & 0.40 \\
\hline 16 & 12-13 & 0.0050 & 0.14 & 0.09 & 0.35 & 0.16 & 0.04 & 0.11 & 0.1 & 0.5 & 0.08 & 0.07 & 0.12 & 0.18 & 0.16 & 0.07 & 0.29 \\
\hline 17 & $12-14$ & 0.0071 & 0.03 & 0.02 & 0.09 & 0.05 & 0.05 & 0.01 & 0.1 & 0.0 & $\underline{2.15}$ & 0.28 & 0.22 & 0.31 & 0.11 & 0.27 & 0.18 \\
\hline 18 & $12-15$ & 0.0045 & 0.08 & 0.11 & 0.28 & 0.15 & 0.23 & 0.02 & 0.4 & 1.4 & 0.19 & 1.25 & 0.85 & 0.11 & 0.11 & 0.17 & 0.27 \\
\hline 19 & 12-16 & 0.0020 & 0.03 & 0.12 & 0.25 & 0.09 & 0.25 & 0.17 & 0.6 & $\overline{0.4}$ & 0.05 & 1.18 & 0.22 & 0.05 & 0.37 & 0.17 & 0.10 \\
\hline 20 & 14-15 & 0.0022 & 0.00 & 0.02 & 0.06 & 0.02 & 0.06 & 0.04 & 0.1 & 0.4 & 1.86 & 0.57 & 0.20 & 0.08 & 0.13 & 0.06 & 0.04 \\
\hline 21 & $\begin{array}{ll}16-17 \\
\end{array}$ & 0.3540 & 0.02 & 0.12 & 0.23 & 0.07 & 0.28 & 0.01 & 0.6 & 0.4 & 0.07 & 0.08 & 0.21 & 0.09 & 0.08 & 0.16 & 0.07 \\
\hline 22 & 15-18 & 0.0045 & 0.03 & 0.06 & 0.14 & 0.05 & 0.14 & 0.01 & 0.3 & 0.4 & 0.07 & $\underline{3.48}$ & 0.03 & 0.05 & 0.10 & 0.07 & 0.05 \\
\hline 23 & 18-19 & 0.0009 & 0.01 & 0.06 & 0.12 & 0.04 & 0.15 & 0.08 & 0.3 & 0.4 & 0.08 & $\overline{1.24}$ & 0.04 & 0.06 & 0.04 & 0.06 & 0.02 \\
\hline 24 & $19-20$ & 0.0004 & 0.03 & 0.07 & 0.10 & 0.02 & 0.19 & 0.08 & $\underline{1.2}$ & 0.2 & 0.08 & 0.25 & 0.05 & 0.00 & 0.02 & 0.06 & 0.05 \\
\hline 25 & $10-20$ & 0.0047 & 0.04 & 0.07 & 0.10 & 0.03 & 0.21 & 0.08 & 0.3 & 0.1 & 0.05 & 0.26 & 0.05 & 0.38 & 0.03 & 0.16 & 0.06 \\
\hline 26 & 10-17 & 0.0009 & 0.04 & 0.12 & 0.19 & 0.02 & 0.34 & 0.16 & 0.6 & 2.4 & 0.04 & 0.27 & 1.22 & 0.22 & 0.04 & 0.15 & 0.01 \\
\hline 27 & $10-21$ & 0.0013 & 0.08 & 0.03 & 0.06 & 0.09 & 0.16 & 0.01 & 0.4 & $\overline{0.2}$ & 0.07 & 0.33 & $\overline{\overline{0.80}}$ & 0.45 & 0.05 & 0.09 & 0.32 \\
\hline 28 & $\begin{array}{ll}10-22 \\
\end{array}$ & 0.0024 & 0.04 & 0.01 & 0.03 & 0.04 & 0.08 & 0.01 & 0.2 & 0.4 & 0.16 & 0.21 & 1.21 & 0.23 & 0.45 & 0.18 & 0.18 \\
\hline 29 & $21-22$ & 0.0010 & 0.01 & 0.01 & 0.03 & 0.01 & 0.02 & 0.01 & 0.3 & 0.1 & 0.11 & 0.28 & 1.03 & 0.01 & 0.30 & 0.22 & 0.16 \\
\hline 30 & $15-23$ & 0.0040 & 0.02 & 0.07 & 0.15 & 0.06 & 0.15 & 0.01 & 0.1 & 0.2 & 0.29 & 0.08 & $\overline{1.98}$ & 0.02 & 0.38 & 0.24 & 0.20 \\
\hline 31 & $22-24$ & 0.0002 & 0.03 & 0.03 & 0.04 & 0.03 & 0.09 & 0.02 & 0.5 & 0.4 & 0.01 & 0.48 & 1.02 & 0.42 & 0.37 & 0.22 & 0.34 \\
\hline 32 & $23-24$ & 0.0041 & 0.01 & 0.07 & 0.13 & 0.06 & 0.16 & 0.12 & 0.1 & 0.4 & 0.32 & $\underline{1.39}$ & 0.86 & 0.63 & 0.67 & 0.24 & 0.18 \\
\hline 33 & $24-25$ & 0.0032 & 0.01 & 0.44 & 0.08 & 0.09 & 0.11 & 0.14 & 0.3 & 0.2 & 0.03 & 0.01 & 0.01 & $\underline{1.23}$ & 0.35 & 0.43 & 0.47 \\
\hline 34 & $25-26$ & 0.0043 & 0.02 & 0.07 & 0.01 & 0.03 & 0.03 & 0.01 & 0.1 & 0.1 & 0.31 & 0.40 & 0.85 & 0.64 & $\underline{1.00}$ & 0.01 & 0.01 \\
\hline 35 & $25-27$ & 0.0036 & 0.02 & 0.04 & 0.07 & 0.01 & 0.14 & 0.14 & 0.3 & 0.2 & 0.03 & 0.00 & $\underline{0.83}$ & 0.64 & 0.02 & 0.48 & 0.46 \\
\hline 36 & $28-27$ & 0.0032 & 0.07 & 0.03 & 0.07 & 0.08 & 0.25 & 0.19 & 0.3 & 0.1 & 0.03 & 0.08 & $\overline{0.86}$ & 0.02 & 1.02 & 0.56 & 0.94 \\
\hline 37 & $27-29$ & 0.0016 & 0.03 & 0.01 & 0.02 & 0.03 & 0.05 & 0.09 & 0.1 & 0.1 & 0.32 & 0.14 & 0.01 & 0.03 & $\underline{2.00}$ & 0.03 & 0.57 \\
\hline 38 & $27-30$ & 0.0035 & 0.03 & 0.01 & 0.02 & 0.03 & 0.05 & 0.01 & 0.1 & 0.3 & 0.01 & 0.02 & 0.01 & 0.01 & 0.06 & 0.04 & $\underline{0.82}$ \\
\hline 39 & $29-30$ & 0.0040 & 0.02 & 0.00 & 0.01 & 0.02 & 0.03 & 0.01 & 0.0 & 0.1 & 0.32 & 0.40 & 0.01 & $\underline{0.63}$ & 0.03 & 0.02 & 0.56 \\
\hline 40 & $8-28$ & 0.0005 & 0.01 & 0.02 & 0.03 & 0.01 & 0.02 & 1.73 & 0.1 & 0.2 & 0.28 & 0.07 & 0.15 & $\overline{0.10}$ & 0.38 & $\underline{2.11}$ & 0.17 \\
\hline 41 & $6-28$ & 0.0008 & 0.03 & 0.05 & 0.09 & 0.05 & 0.21 & 1.61 & 0.2 & 0.1 & 0.61 & 0.34 & 0.69 & 0.54 & 1.6 & $\underline{8.54}$ & 0.78 \\
\hline
\end{tabular}

Table 9: Compare the pre-fault \& post fault line current magnitude in p.u. of each line of IEEE 30 Bus system: 


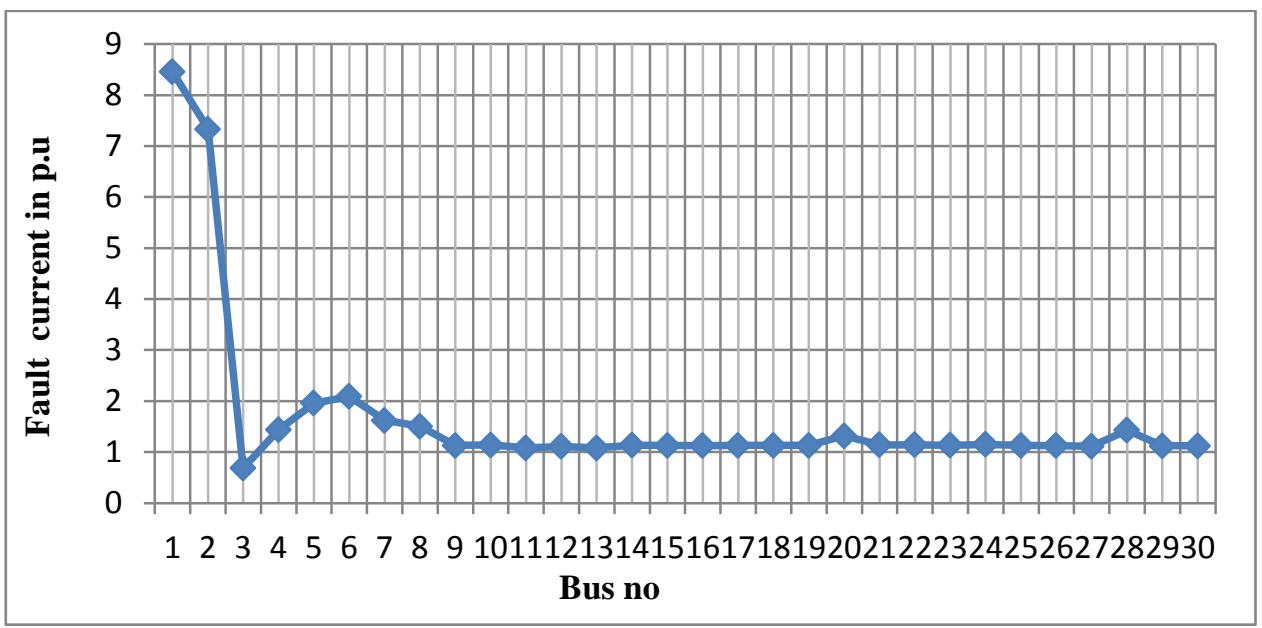

Figure 8: Graphical Representation for Current flow in line no.1 when there is a fault occurs on IEEE 30 bus

Table no.9 represents the comparison of line flow currents during LLL fault with the pre-fault line flow currents; it is analysing that line currents increases from their normal value when there is a fault at different buses.

- $\quad$ Table 10: SCMVA and Circuit Breaker Ratings in each line of the IEEE 30 bus system:

\begin{tabular}{|c|c|c|}
\hline Line no. & SCMVA & Circuit breaker rating (MVA) \\
\hline 1 & 895.7 & 900 \\
\hline 2 & 398.5 & 400 \\
\hline 3 & 348.4 & 350 \\
\hline 4 & 1060.8 & 1070 \\
\hline 5 & 393.9 & 400 \\
\hline 6 & 354.5 & 360 \\
\hline 7 & 870.62 & 880 \\
\hline 8 & 365.62 & 370 \\
\hline 9 & 379.0 & 380 \\
\hline 10 & 1070 & 1070 \\
\hline 11 & 387.5 & 390 \\
\hline 12 & 146.58 & 150 \\
\hline 13 & 184.0 & 190 \\
\hline 14 & 268.51 & 270 \\
\hline 15 & 189.5 & 190 \\
\hline 16 & 60.72 & 70 \\
\hline 17 & 212.6 & 220 \\
\hline 18 & 152.8 & 160 \\
\hline 19 & 186.7 & 190 \\
\hline 20 & 191.9 & 200 \\
\hline 21 & 124.8 & 130 \\
\hline 22 & 357.7 & 360 \\
\hline 23 & 124.4 & 130 \\
\hline 24 & 123.5 & 130 \\
\hline 25 & 248.08 & 250 \\
\hline 26 & 122.48 & 130 \\
\hline 27 & 248.2 & 250 \\
\hline 28 & 156.9 & 160 \\
\hline 29 & 248.2 & 250 \\
\hline 30 & 198.79 & 200 \\
\hline 31 & 144.4 & 150 \\
\hline 32 & 139.5 & 140 \\
\hline 33 & 124.7 & 130 \\
\hline 34 & 101.6 & 110 \\
\hline 35 & 83.3 & 90 \\
\hline 36 & 103.6 & 110 \\
\hline 37 & 203.2 & 210 \\
\hline 38 & 81.6 & 90 \\
\hline 39 & 63.8 & 70 \\
\hline 40 & 213.9 & 220 \\
\hline 41 & 865.9 & 870 \\
\hline
\end{tabular}

From Table 10, in line no. 1 maximum current flowing when there is a fault occur at bus no. 1 i.e. 8.458 p.u \& in line no. 2 maximum current flowing when there is a fault at bus no.1.\& in line no. 3 maximum current flowing during fault at bus no. 4 \& in line no. 4 maximum current flowing when there is a fault at bus no. 3 \& similarly check other lines for fault at each bus of the system and choose the SCMVA rating of $\mathrm{CB}$ for each line acc. to above table. This effect is cleared by a graph that showing the current flow in line no. 1 when fault occur at different buses, as shown in fig.8.

From table 10 it can be observed that transmission line no. 10 having a higher fault current $\&$ higher circuit breaker rating i.e. 1070MVA. And also line no flows higher fault current. And line 16 is less affected.

\section{CONCLUSION}

The evaluation of Fault analysis or short circuit analysis is very important part of power system analysis for stable and economical operations of a Power System network. The Short circuit studies and hence the fault analyses is are very important for the power system studies since they provide data such as voltages and currents during and after the various types of faults which are necessary in designing the protective schemes of the power system. The main task is to calculate fault conditions and provide protective equipments designed to isolate the faulted zone from the remainder of the system in the appropriate time. The simulation is take place on symmetrical fault (LLL) on different buses of a power system network and to estimate the state of the power system before and after a fault, which includes various bus voltages and current flow on various transmission lines. From above results, it is seen that during short circuit fault (LLL) voltage magnitude at faulty buses reduced to zero and current flow in the lines increases. During LLL fault In IEEE 6 bus system, line no. 4 is most affected i.e. in line no. 4 maximum fault current flows during fault at bus no. 4 and in IEEE 30 bus system, line no. 10 is most effected when there is faults occur at bus no.8. So according to the values of fault current flows in the lines, circuit breaker rating is chosen.). It is concluded that in the lines of 6 bus system 
circuit breaker rating ranges within 210 MVA and 530MVA and in the lines of 30 bus system circuit breaker rating ranges within 70MVA and $1070 \mathrm{MVA}$.

\section{APPENDIX}

APPENDIX - 1: Test System Data of IEEE 6 bus system: Fault analysis has been done on standard IEEE 6 bus systems, and it consists of 6 buses and 7 lines. Line data and load data for IEEE 6 bus system is given below.

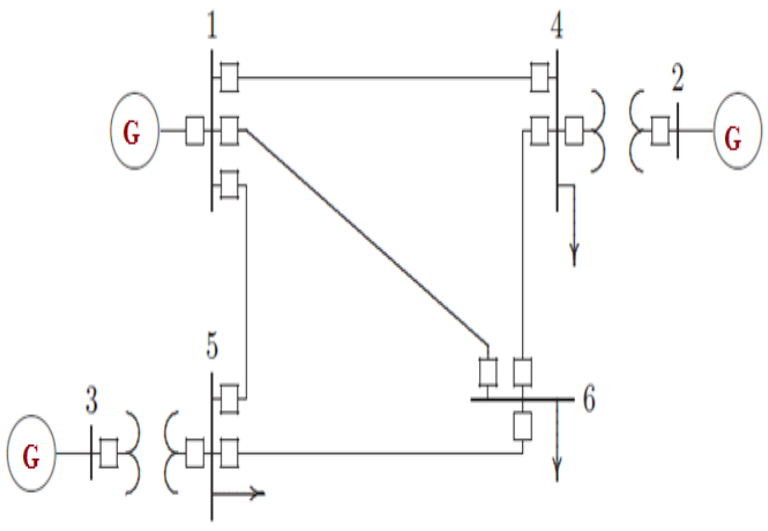

Fig 9: IEEE 6 bus system

- $\quad$ Line data for IEEE 6 bus system:

\begin{tabular}{|l|l|l|l|l|}
\hline $\begin{array}{l}\text { Line } \\
\text { no. }\end{array}$ & $\begin{array}{c}\text { From } \\
\text { To bus }\end{array}$ & $\begin{array}{l}\text { Resistance, } \\
\text { R(p.u) }\end{array}$ & $\begin{array}{l}\text { Reactance, } \\
\text { X(p.u) }\end{array}$ & $\begin{array}{l}\text { Half line } \\
\text { charging } \\
\text { Susceptance, } \\
1 / 2 \text { B(p.u) }\end{array}$ \\
\hline 1 & $1-4$ & 0.035 & 0.225 & 0.0065 \\
\hline 2 & $1-5$ & 0.025 & 0.105 & 0.0045 \\
\hline 3 & $1-6$ & 0.04 & 0.215 & 0.005 \\
\hline 4 & $2-4$ & 0.00 & 0.035 & 0.000 \\
\hline 5 & $3-5$ & 0.00 & 0.042 & 0.000 \\
\hline 6 & $4-6$ & 0.028 & 0.125 & 0.0035 \\
\hline 7 & $5-6$ & 0.026 & 0.175 & 0.030 \\
\hline
\end{tabular}

- $\quad$ Load data for IEEE 6 bus system:

\begin{tabular}{|l|l|l|l|l|l|l|}
\hline \multirow{2}{*}{$\begin{array}{l}\text { Bus } \\
\text { no }\end{array}$} & \multicolumn{2}{|c|}{ Bus Voltage } & \multicolumn{2}{c|}{ Load (p.u) } & \multicolumn{2}{c|}{ Generation (p.u) } \\
\cline { 2 - 7 } & $\begin{array}{l}\text { Voltage } \\
\text { magnitud } \\
\text { e (p.u) }\end{array}$ & $\begin{array}{l}\text { Angle } \\
\text { (rad) }\end{array}$ & $\begin{array}{l}\text { MW } \\
(\mathbf{L})\end{array}$ & $\begin{array}{l}\text { Mvar } \\
(\mathbf{L})\end{array}$ & $\begin{array}{l}\text { MW } \\
(\mathbf{G})\end{array}$ & $\begin{array}{l}\text { Mvar } \\
(\mathbf{G})\end{array}$ \\
\hline 1 & 1.06 & 0.00 & 0.00 & 0.00 & 0.00 & 0.00 \\
\hline 2 & 1.04 & 0.00 & 150.0 & 0.00 & 0.00 & 0.00 \\
\hline 3 & 1.03 & 0.00 & 100.0 & 0.00 & 0.00 & 0.00 \\
\hline 4 & 1.0 & 0.00 & 100.0 & 70.00 & 0.00 & 0.00 \\
\hline 5 & 1.0 & 0.00 & 90.00 & 30.0 & 0.00 & 0.00 \\
\hline 6 & 1.0 & 0.00 & 160.0 & 110.0 & 0.00 & 0.00 \\
\hline
\end{tabular}

APPENDIX - 2: Test System Data of IEEE 30 bus system:

Fault analysis has been done on standard IEEE 30 bus systems, and it consists of 30 buses and 41 lines. Line data and load data for IEEE 30 bus system is given below.

Fig .10: IEEE 30 bus system

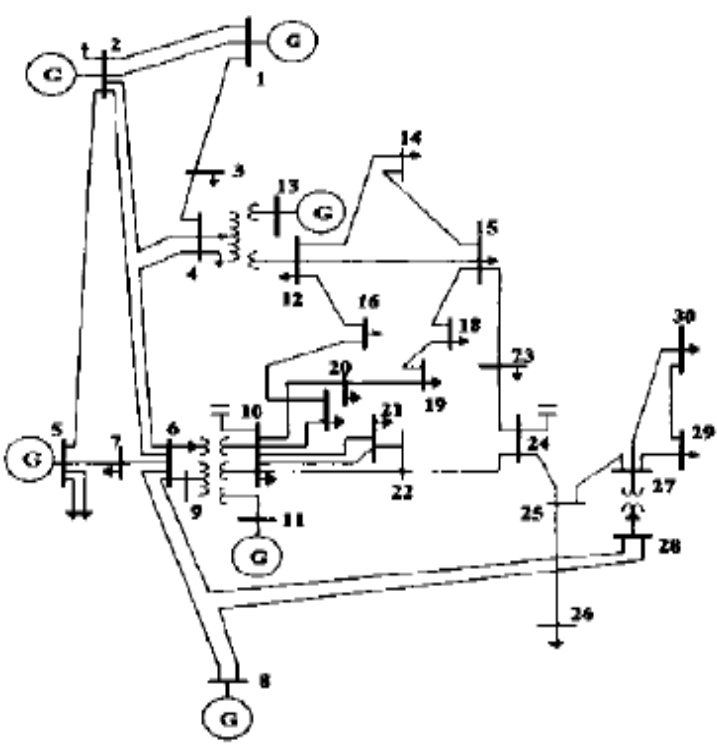

- Line data for IEEE 30 bus system:

\begin{tabular}{|c|c|c|c|c|}
\hline $\begin{array}{l}\text { Line } \\
\text { no. }\end{array}$ & $\begin{array}{l}\text { From } \\
- \\
\text { To }\end{array}$ & $\begin{array}{l}\text { Resistance } \\
\text { R (p.u) }\end{array}$ & $\begin{array}{l}\text { Reactance } \\
\text { X(p.u) }\end{array}$ & $\begin{array}{l}\text { Half line } \\
\text { charging } \\
\text { Susceptance } \\
\frac{1}{2} \text { B (p.u) }\end{array}$ \\
\hline 1 & $1-2$ & 0.0192 & 0.0575 & 0.0264 \\
\hline 2 & $1-3$ & 0.0452 & 0.1652 & 0.0204 \\
\hline 3 & $2-4$ & 0.057 & 0.1737 & 0.0184 \\
\hline 4 & $3-4$ & 0.0132 & 0.0379 & 0.0042 \\
\hline 5 & $2-5$ & 0.0472 & 0.1983 & 0.0209 \\
\hline 6 & $2-6$ & 0.0581 & 0.1763 & 0.0187 \\
\hline 7 & $4-6$ & 0.0119 & 0.0414 & 0.0045 \\
\hline 8 & $5-7$ & 0.046 & 0.116 & 0.0102 \\
\hline 9 & $6-7$ & 0.0267 & 0.082 & 0.0085 \\
\hline 10 & $6-8$ & 0.012 & 0.042 & 0.0045 \\
\hline 11 & $6-9$ & 0 & 0.208 & 0 \\
\hline 12 & $6-10$ & 0 & 0.556 & 0 \\
\hline 13 & $9-11$ & 0 & 0.208 & 0 \\
\hline 14 & $9-10$ & 0 & 0.11 & 0 \\
\hline 15 & $4-12$ & 0 & 0.256 & 0 \\
\hline 16 & $12-13$ & 0 & 0.14 & 0 \\
\hline 17 & $12-14$ & 0.1231 & 0.2559 & 0 \\
\hline 18 & $12-15$ & 0.0662 & 0.1304 & 0 \\
\hline 19 & $12-16$ & 0.0945 & 0.1987 & 0 \\
\hline 20 & 14-15 & 0.221 & 0.1997 & 0 \\
\hline 21 & $16-17$ & 0.0524 & 0.1923 & 0 \\
\hline 22 & $15-18$ & 0.1073 & 0.2185 & 0 \\
\hline 23 & $18-19$ & 0.0639 & 0.1292 & 0 \\
\hline 24 & $19-20$ & 0.034 & 0.068 & 0 \\
\hline 25 & $10-20$ & 0.0936 & 0.209 & 0 \\
\hline 26 & $10-17$ & 0.0324 & 0.0845 & 0 \\
\hline 27 & $10-21$ & 0.0348 & 0.0749 & 0 \\
\hline 28 & $10-22$ & 0.0727 & 0.1499 & 0 \\
\hline 29 & $21-22$ & 0.0116 & 0.0236 & 0 \\
\hline 30 & $15-23$ & 0.1 & 0.202 & 0 \\
\hline 31 & $22-24$ & 0.115 & 0.179 & 0 \\
\hline 32 & $23-24$ & 0.132 & 0.27 & 0 \\
\hline 33 & $24-25$ & 0.1885 & 0.3292 & 0 \\
\hline 34 & $25-26$ & 0.2544 & 0.38 & 0 \\
\hline 35 & $25-27$ & 0.1093 & 0.2087 & 0 \\
\hline 36 & $28-27$ & 0 & 0.396 & 0 \\
\hline 37 & $27-29$ & 0.2198 & 0.4153 & 0 \\
\hline 38 & $27-30$ & 0.3202 & 0.6027 & 0 \\
\hline 39 & $29-30$ & 0.2399 & 0.4533 & 0 \\
\hline 40 & $8-28$ & 0.0636 & 0.2000 & 0.0214 \\
\hline 41 & $6-28$ & 0.0169 & 0.0599 & 0.065 \\
\hline
\end{tabular}

- Load data for IEEE 30 Bus system: 
Vol. 3, Issue 8, August 2015

\begin{tabular}{|c|c|c|c|c|c|c|}
\hline \multirow[b]{2}{*}{$\begin{array}{l}\text { Bus } \\
\text { no }\end{array}$} & \multicolumn{2}{|l|}{ Bus voltage } & \multicolumn{2}{|l|}{ Load } & \multicolumn{2}{|c|}{ Generation } \\
\hline & $\begin{array}{l}\text { Voltage } \\
\text { magnitude } \\
\text { (p.u) }\end{array}$ & $\begin{array}{l}\text { Angle } \\
\text { (rad) }\end{array}$ & $\begin{array}{l}\text { MW } \\
\text { (L) }\end{array}$ & $\begin{array}{l}\text { Mvar } \\
\text { (L) }\end{array}$ & $\begin{array}{l}\text { MW } \\
\text { (G) }\end{array}$ & $\begin{array}{l}\text { Mvar } \\
\text { (G) }\end{array}$ \\
\hline 1 & 1.06 & 0.00 & 0.00 & 0 & 0 & 0 \\
\hline 2 & 1.045 & 0.00 & 21.7 & 12.7 & 40 & 0 \\
\hline 3 & 1.000 & 0.00 & 2.4 & 1.2 & 0 & 0 \\
\hline 4 & 1.060 & 0.00 & 7.6 & 1.6 & 0 & 0 \\
\hline 5 & 1.010 & 0.00 & 94.2 & 19 & 20 & -40 \\
\hline 6 & 1.000 & 0.00 & 0.00 & 0 & 0 & 0 \\
\hline 7 & 1.000 & 0.00 & 22.8 & 10.9 & 0 & 0 \\
\hline 8 & 1.010 & 0.00 & 30 & 30.6 & 20 & -10 \\
\hline 9 & 1.000 & 0.00 & 0.00 & 0 & 0 & 0 \\
\hline 10 & 1.000 & 0.00 & 5.8 & 2 & 0 & 0 \\
\hline 11 & 1.082 & 0.00 & 0.00 & 20 & 0 & -6 \\
\hline 12 & 1.000 & 0.00 & 11.2 & 7.5 & 0 & 0 \\
\hline 13 & 1.071 & 0.00 & 0.00 & 20 & 0 & -6 \\
\hline 14 & 1.000 & 0.00 & 6.2 & 1.6 & 0 & 0 \\
\hline 15 & 1.000 & 0.00 & 8.2 & 2.5 & 0 & 0 \\
\hline 16 & 1.000 & 0.00 & 3.5 & 1.8 & 0 & 0 \\
\hline 17 & 1.000 & 0.00 & 9 & 5.8 & 0 & 0 \\
\hline 18 & 1.000 & 0.00 & 3.2 & 0.9 & 0 & 0 \\
\hline 19 & 1.000 & 0.00 & 9.5 & 3.4 & 0 & 0 \\
\hline 20 & 1.000 & 0.00 & 2.2 & 0.7 & 0 & 0 \\
\hline 21 & 1.000 & 0.00 & 17.5 & 11.2 & 0 & 0 \\
\hline 22 & 1.000 & 0.00 & 0.00 & 0.00 & 0 & 0 \\
\hline 23 & 1.000 & 0.00 & 3.2 & 1.6 & 0 & 0 \\
\hline 24 & 1.000 & 0.00 & 8.7 & 6.7 & 0 & 0 \\
\hline 25 & 1.000 & 0.00 & 0.00 & 0.00 & 0 & 0 \\
\hline 26 & 1.000 & 0.00 & 3.5 & 2.3 & 0 & 0 \\
\hline 27 & 1.000 & 0.00 & 0.00 & 0.00 & 0 & 0 \\
\hline 28 & 1.000 & 0.00 & 0.00 & 0.00 & 0 & 0 \\
\hline 29 & 1.000 & 0.00 & 2.4 & 0.9 & 0 & 0 \\
\hline 30 & 1.000 & 0.00 & 10.6 & 1.9 & 0 & 0 \\
\hline
\end{tabular}

\section{REFERENCES}

[1] Teng Jen-Hao."Systematic short circuit analysis Method for unbalanced distribution systems", IEE ProcGeneration,Transm Distribut 2005;4:549-55.

[2] Gil BRV. "Application of the generalized Thevenin's Theorem for solving asymmetrical series faults", In Proceedings first IEEE international conference, $12-14$ 1995. p. 271-5.

[3] Strezoski VC, Bekut DD. "A canonical model for the Study of faults in power systems”, IEEE Trans Power System 1991;6(4):1493-9.

4] Dzafic.i , Siemens AG . "Impedance based Fault Location for weakly meshed distribution networks", IEEE Trans Power System 2011 ;110-10-9.

[5] Jawad Talaq . "Fault calculations using three terminal Thevenin's equivalent circuit", Electrical Power and Energy Systems 33 (2011);1462-1469.

[6] Han,Z.X, "Generalized Method of Analysis of Simultaneous Faults in Electric Power System”, ,IEEE Trans Power System 1982;PAS-101.

[7] Martín C. Rodríguez Paz, Renato G. Ferraz , Arturo Suman Bretas "System unbalance and fault Impedance effect on faulted distribution networks" Electrical Power and Energy Systems (2011);1462-69.

[8 ]Andre , D. Filomena , Mariana Resener , Rodrigo H.Salim , "Distribution systems fault analysis Considering fault resistance Estimation" , Electrical Power and Energy Systems 33 (2011) 1462-1469.

[9] E.B. Makram, M.A. Bou-Rabee,"Three-phase Modelling of unbalanced distribution systems during open/ shunt fault Conditions using the bus impedance matrix", Electric. Power Systems Research (1987) 173_83.

[10]Gerardus C.Paap,"Symmetrical component in the time domain and their application to power network calculation," IEEE Transaction on Power System, vol.15, May 2000,p.p: 522-528

[11]Shengli Huang, Ruihua Song, Xiaoxin Zhou, "Analysis of balanced \& Unbalanced Fault in power system using dynamic Phasors", IEEE paper on power system technology, vol. 3, 2002, p.p. 155057.
[12] Zhang Chen, Z hou Lixing, " $220 \mathrm{KV}$ transmission line fault diagnosis and analysis", IEEE Transaction on Power System , Jan 2012,p.p. 1343-45.

[13] Teng JH,"Systematic short-circuit-analysis method for unbalanced distribution systems". IEE Proc Gener Transm Distrib 2005;152(4):549-55

[14] Abdel-Akher M, Nor K, "Fault analysis of multiphase distribution systems using symmetrical components". IEEE Trans Power 2010;25(4):2931-9

[15] Mao Y, Miu K, "Radial distribution system short circuit analysis with lateral and load equivalencing: solution algorithms and numerical results", IEEE power engineering summer meeting, 2000, 449-53

[16] Teng JH, "Fast short circuit analysis method for unbalanced distribution systems', In IEEE power engineering society general meeting, 2003, 240-5

[17] Akhilesh Mathur,Vinay Pant, Biswarup Das, “Unsymmetrical shortcircuit analysis for distribution system considering loads", Electrical Power and Energy Systems, 70(2015), p.p 27-28,

[18]Kalantari A, Kouhsari SM, "An exact piecewise method for fault studies in interconnected networks". Int J Electr Power Energy Syst 2008;30(3):216-25.

[19] Abouelenin FM, “ A complete algorithm to fault calculation due to simultaneous faults Combination of short circuits and open lines". In: IEEE technical conference; 2002. p. 522-6.

[20] Gross CA, "Fault calculations in power systems subject to multiple faults". In: Proceedings of the 22nd symposium on system theory; 11-16

[21] MaLiao Y, "Fault location for single-circuit line based on busimpedance matrix utilizing voltage Measurements", IEEE Trans Power Delivery 2008;23(2):609-17.rch 1990. p. 598-601.

[22] Halpin SM, Grigsby LL, Gross CA, Nelms RM. "An improved fault analysis algorithm for unbalanced multi-phase power distribution systems". IEEE Trans Power Deliv 1994;9(3):1332-1340

[23] Ravi Kumar Tiwari, Nidhi Singh, Ambarish Kumar, D Suresh Babu, "Analysis of Symmetrical Fault with Fault Impedance in Power System", Global Research Analysis, Volume: 2, Issue:1, January 2013

[24] Hadi Saadat, "Power System Analysis", Tata McGraw Hill Publication, 2009

25] I.J. Nagrath, D.P.Kothari, Modern Power System Analysis, Tata McGraw-Hill Publication, 2012 